\title{
Dynamics of electronic excitations in YBCO at low temperatures
}

\author{
I. Ya. Fugol, V. N. Samovarov, and M. Yu. Libin \\ B. Verkin Institute for Low Temperature Physics and Engineering, National Academy of Sciences of \\ Ukraine, Lenin Ave. 47, Kharkov, 310164, Ukraine \\ E-mail: fugol@ilt.kharkov.ua
}

Receved November 11, 1998, revised December 24, 1998

\begin{abstract}
We present the results of low-temperature optical experiments (absorption and reflection) in visible and middle infrared frequency regions with epitaxial films of copper oxide materials like a $\mathrm{Y}_{1} \mathrm{Ba}_{2} \mathrm{Cu}_{3} \mathrm{O}_{6+x}$. Based on our data and reference data, we consider the anomalous spectral effects in HTSC having no analogy with conventional BCS superconductors: (1) the optical response to superconducting transition at $T_{c}$; (2) the spectral weight redistribution induced by chemical doping and temperature; (3) the drastic enhancement of low-temperature photodoping; (4) the long spin-structure relaxation via temperature variations seen in the optical spectra. The thorough analysis of the results obtained is fully compatible with the concept of two-component system of light and heavy carriers (holes), being in dynamical coexistence with each other. The dynamical coexistence of the intraband carriers occurs on the background of strongly correlation interrelation of the heavy quasiparticles with the optical interband (charge transfer) excitations.
\end{abstract}

PACS: 74.72.Bk

\section{Introduction}

In recent years the optical spectroscopy of HTSC at frequencies much higher than the superconducting gap, $\hbar \omega>>\Delta$, has been found to be informative for understanding the nature of high- $T_{c}$ superconductivity, in particular, for studying the anomaly of the normal-state properties of HTSC. With regards to room temperature $(R T)$ optical experiments, the following distinct features of the HTSC spectra, which were originally associated with the unusual electron structure, deserve mention: (i) the pronounced optical effect of strongly correlated electrons (holes) under doping, manifesting itself in spectral weight redistribution between the high-energy (interband) region of spectra and the low-energy (intraband) one; (ii) the declination of the optical conductivity from the Drude-like behavior (standard for free carriers) and the appearance of an additional band in the middle infrared region; (iii) the inherent potentiality of photodoping, which results in pumping of carriers under irradiation with light quanta at the frequencies of interband charge transfer transitions above the optical gap $\hbar \omega>\hbar \omega_{g} \cong 1.6 \mathrm{eV}$; (iv) the optical relaxation of the reflection coefficient in the course of aging and the oxygen ordering of HTSC samples after cooling from high temperature $(T \leq 450 \mathrm{~K})$ to RT.

The mentioned important results for different HTSC are presented, for example, in [1-6]. All the above features were measured in the optical range from $10^{-2}$ to $5 \mathrm{eV}$ with different doping regimes of $\mathrm{Y}_{1} \mathrm{Ba}_{2} \mathrm{Cu}_{3} \mathrm{O}_{6+x}$ : the dielectric phase, $x<0.3$; the strange metal phase at underdoping, $0.35<x<0.8$; the metal at optimal doping, $x \approx 0.9$; the overdoped metal with depressed superconductivity, $x>0.95$. Because there are two different cuprate structures in the $a b$ geometry of $\mathrm{Y}_{1} \mathrm{Ba}_{2} \mathrm{Cu}_{3} \mathrm{O}_{6+x}-\mathrm{CuO}_{2}$ (active plane and $\mathrm{CuO}_{x}$ (chain structure along the $b$ axis), it is essential that the contributions from these planes to absorption and reflection should be separated in experiments with light polarization. This can be most easily done in polarization experiments with $E \| a$ and $E \| b$ on untwinned crystals. In this respect most intriguing are the data of polarization spectrum measurements on untwinned single crystals of $\mathrm{YBaCuO}$ for $a$ and $b$ axes [2,6]. For example, the optical conductivity spectrum of $\mathrm{Y}_{1} \mathrm{Ba}_{2} \mathrm{Cu}_{3} \mathrm{O}_{6+x}$ for different doping amounts at RT according [2] is shown in Fig. 1. Below the energy of the charge transfer optical gap, $E \cong 1.6 \mathrm{eV}$, separating the 


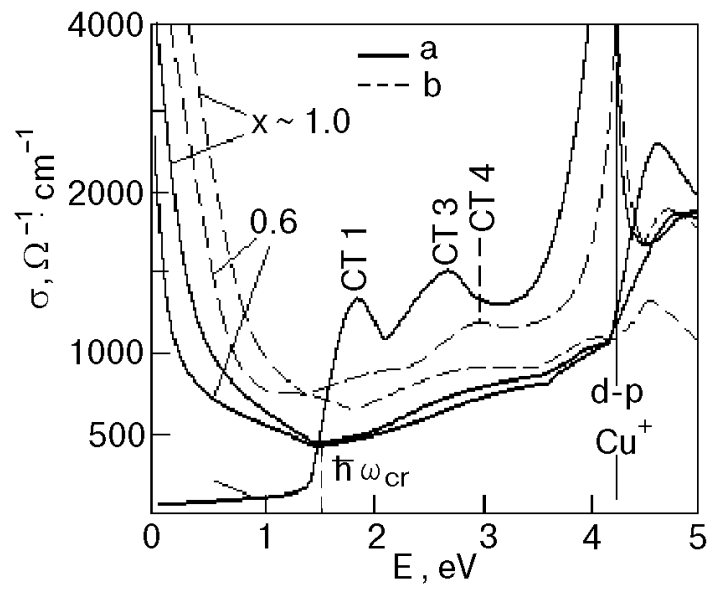

Fig. 1. Optical conductivity of untwinned YBCO crystals for various composition $x$ at RT [2]: along $a$ axis (-); along $b$ axis $(---)$.

lower valent and the upper Hubbard bands there are intraband transitions. Above this energy, $E>E_{g}$, one can observe interband absorption bands with charge transfer from $\mathrm{O}^{2-}$ to $\mathrm{Cu}^{2+}-\mathrm{CT} 1, \mathrm{CT} 3, \mathrm{CT} 4$ which are most prominent at low doping. Still higher in energy, at $E=4.1 \mathrm{eV}$, there is a band which belongs to the transition $(d-p)$ in the local center of $\mathrm{Cu}^{+}$of $\mathrm{CuO}_{x}$ plane. Figure 1 clearly shows the evolution of optical conductivity spectra with doping from the dielectric to a superconducting state. The correlation effect in the spectra with doping is revealed in the form of integral spectral weight redistribution from the interband charge transfer (CT) transitions in the visible (VIS) region to the intraband transitions in the middle infrared one (MIR). The spectral change indicates that the conduction and valence band states of YBCO insulator are redistributed by doping to construct new states which give rise to low-energy excitations below crossover point $\hbar \omega_{c r}$. As seen from Fig. 1, there is the crossover point $\omega_{c r}$ at which $\sigma_{1}(\omega)$ is constant with doping. It should be mentioned that in general the existence of the doping-independent crossover point $\omega_{\text {cr }}$ suggests that the optical gap in $\mathrm{Y}_{1} \mathrm{Ba}_{2} \mathrm{Cu}_{3} \mathrm{O}_{6+x}$ is not generated by the charge density wave (the spectrum of optical excitations associated with the charge density wave shifts dramatically to a red region as a whole on doping). The optical spectra of $\mathrm{YBaCuO}$ in the vicinity of intraband transitions have a Drude peak, centered at $\omega=0$ with width of $1 / T$ and additional spectral weight in the MIR region (with the MIR maximum at $0.6 \mathrm{eV}$ ). It is the occurrence of additional absorption in the MIR region that results in the difference of the frequency dependence $\sigma(\omega)$ from the law $\sigma(\omega) \sim \omega^{-2}$ expected for the ordinary Fermi liquid.
And that once entailed the need for development of new concepts of HTSC electronic spectrum (for instance, the concept of «marginal» and «nesting» Fermi-liquid [7]). It should be emphasized that in conventional BCS-superconductors no radically new optical effect were observed at low temperatures (LT) as compared to RT in the region $\hbar \omega>>\Delta$, moreover, no optical response to the emergence of the SC state was detected. In the case of HTSC the LT dynamics of charges displays new effects which would signaled on precursory processes to superconductivity.

This paper presents the experimental results of later years on some nonordinary spectral properties of the normal phase of $\mathrm{Y}_{1} \mathrm{Ba}_{2} \mathrm{Cu}_{3} \mathrm{O}_{6+x}$ in VIS and MIR, revealed at different regimes of doping and temperature variations within the LT range below $T=200 \mathrm{~K}$ down to $20 \mathrm{~K}$. In the main these are the data of investigations under the Program of the National Academy of Sciences of Ukraine. All optical measurements were carried out on thin epitaxial films $l \approx 2000-3000 \AA$ in thickness oriented parallel to the $a b$ plane. The films were grown in German scientific centers (University of Erlangen; Munich Technical University). The films were tested for their electrical, magnetic and structure characteristics. Some details of the LT optical measurments were described in [8].

At present it can be said with confidence that the hole subsystem dynamics in HTSC materials is largely determined by the Fermi-liquid effects and the strong correlation interaction between intraand interband transitions. However, up to now the correlation redistribution along spectra has been studied only for the doping effect at RT and has not been considered for other processes of charge dynamics. According to the data [8] and the results presented here, the integral redistribution of spectral weight in HTSC also occurs in experiments with temperature variations, in photoinduced spectra, in experiments with provocation of spin-structure instability and so on. Therefore, the LT spectral data should be analyzed from the general viewpoint. We would like to stress that all LT features in the MIR and VIS spectra can be naturally examined in terms of the Mott-Hubbard model of $2 D$ strongly correlated electrons with taking into account the intense spin fluctuations at LT.

\section{Optical response to SC transition at $T_{c}$ and temperature dependences of spectral functions}

One of the unexpected spectroscopic results in copper oxides was the finding of sensitivity to the 

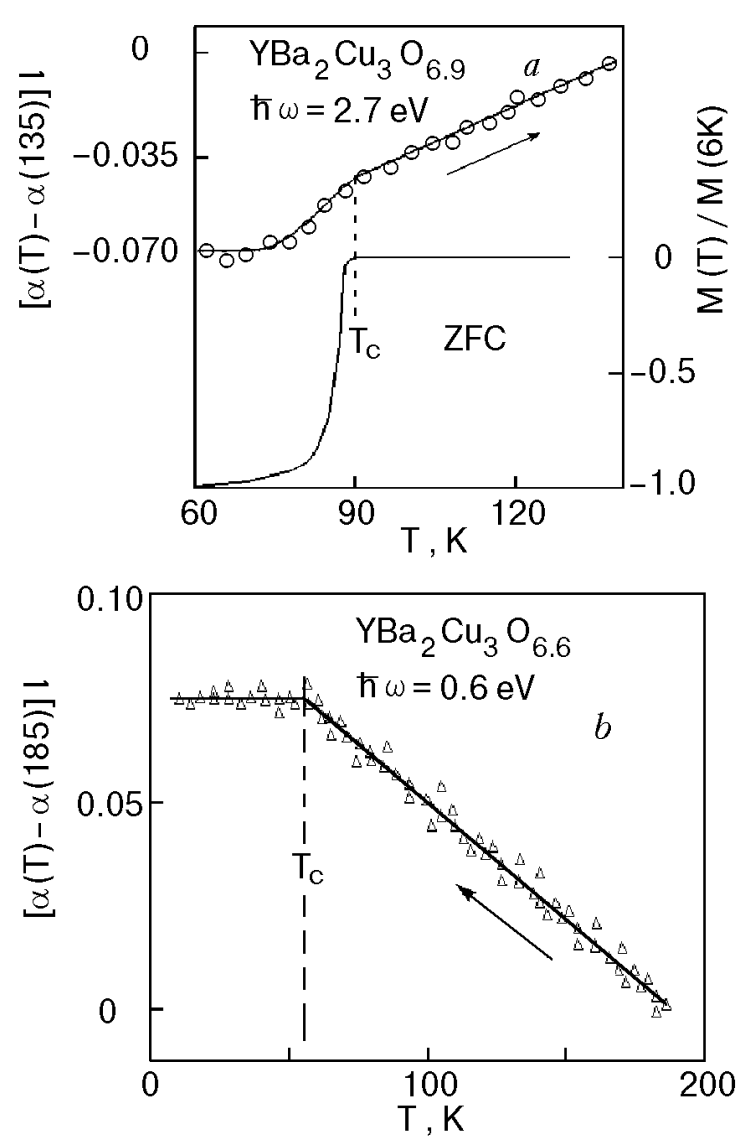

Fig. 2. Temperature dependences of the differential absorption coefficient for VIS (a) and MIR (b) with different $x$ (in (a) shown are magnetic measurements of the film for ZFC regime also).

onset of superconductivity for the transmitted electromagnetic field of optical frequencies $\hbar \omega>>\Delta$. The optical response in absorption and reflection to $T_{c}$ was detected both in VIS and MIR [9-12]. The subsequent experiments confirmed the occurrence of this peculiar effect in $\mathrm{YBaCuO}$ and other HTSC in a wide range of doping. It was shown that the response arose under optical transitions belonging to the conducting plane of $\mathrm{CuO}_{2}$. The $T$ dependence of the differential absorption coefficient at fixed frequency $\Delta \alpha_{\omega}(T) l=\left[\alpha_{\omega}(T)-\alpha_{\omega}\left(T_{0}\right)\right] l$ (for the CT maxima at $2.6 \mathrm{eV}$ ) is shown in Fig. 2, $a$ and that for the differential absorption coefficient of the MIR maximum at $0.6 \mathrm{eV}$ is shown in Fig. 2,b. As it can be seen, the $T_{c}$ point coincides closely with the the kink of the functions $\alpha_{\omega}(T)$. Note that the optical response to the $\mathrm{SC}$ transition in HTSC was also detected in luminescence spectra on oxygen $F$ centers at frequencies near 2-3 eV [13].

The temperature behavior of the optical spectra in $\mathrm{YBaCuO}$ was found to be uncommon. Below $T_{c}$ one can observe a continuos freezing of the $\stackrel{c}{T}$ dependences. Particularly striking is the $T$ behavior of $\alpha_{\omega}(T)$ and $R_{\omega}(T)$ for the normal state at $T>T_{c}$ : for the regimes of underdoping and optical doping the $T$ dependences of the absorption and reflection coefficients have different signs in the VIS and MIR region: both $\alpha_{\omega}(T)$ and $R_{\omega}(T)$ are increasing functions of $T$ in VIS and they are decreasing ones of $T$ in MIR (see Fig. 2, $a, b$ and $[8,10]$ ). However, in the overdoping regime $(x=0.95)$ the CT transitions in VIS undergo fundamental changes with temperature and the VIS spectral function $(T)$ measured decreases with temperature similar to the MIR one [8]. It is like that the correlation spectral weight redistribution is terminated in the overdoping regime where $\mathrm{YBaCuO}$ becomes an uncorrelated metal, in which the upper and lower Hubbard bands are merged together.

Take note that the dependences $\alpha_{\omega}(T)$ and $R_{\omega}(T)$ have no selective character in frequency and the $T$ changes touch on the whole wide region in the VIS and MIR spectra. The differential absorption spectra $\Delta \alpha(\omega) l=\left(\alpha f(\omega)-\alpha^{i}(\omega)\right) l$ (where $f$ and $i$ mark the final and initial conditions of the measurements) are shown in Fig. 3, $a$ for doping and in Fig. 3,b for cooling from 250 to $80 \mathrm{~K}$ (for comparison). In both cases one can observe a decrease in the integral spectral weight of the CT absorption in
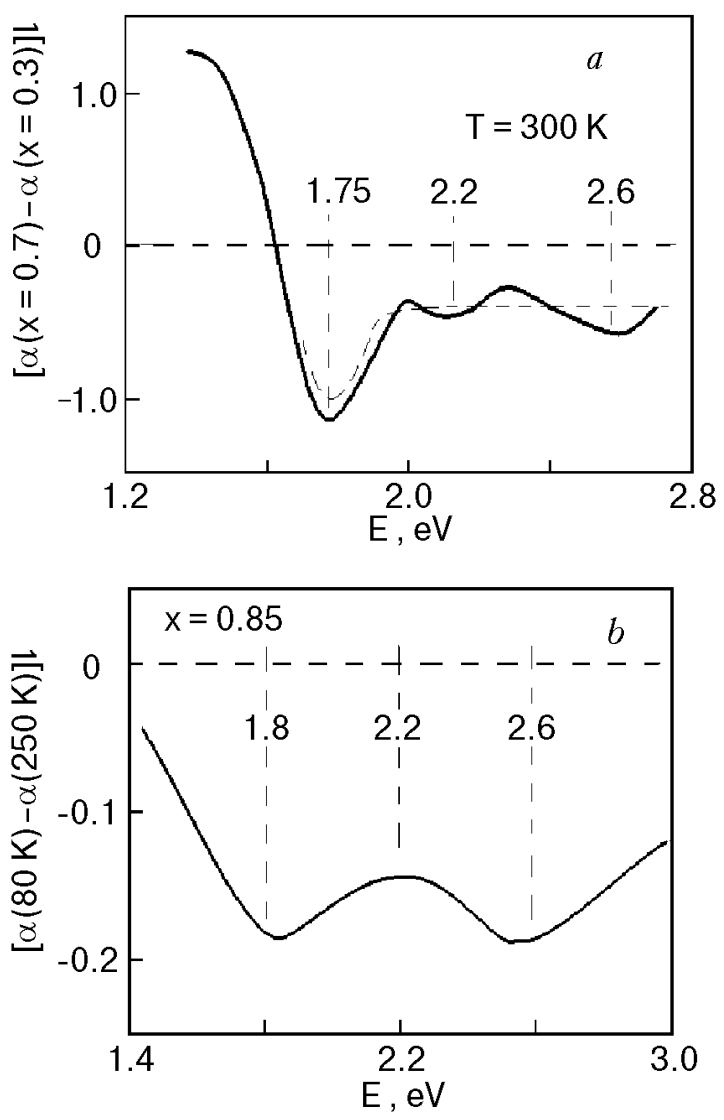

Fig. 3. The differential absorption spectra in YBCO in the CT interband region of frequencies: on doping from $x=0.3$ to 0.7 at RT (a); on cooling from $T=250 \mathrm{~K}$ to $80 \mathrm{~K}$ for $x=0.85(b)$. 


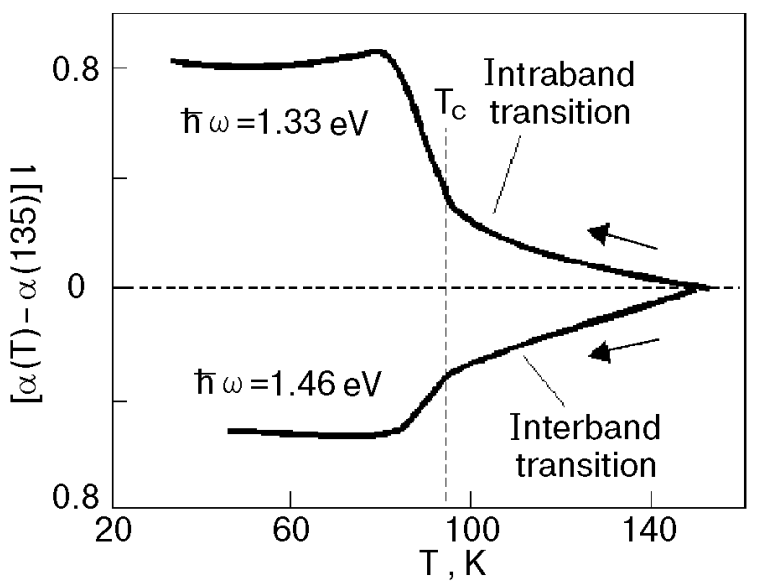

Fig. 4. Temperature dependences of the differential absorption coefficient for two frequences in the vicinity of the crossover point (the upper curve for $\hbar \omega<\hbar \omega_{\text {cr }}$, the lower curve for $\left.\hbar \omega>\hbar \omega_{\mathrm{cr}}\right)$.

favour of the MIR absorption under cooling as well as under doping (but the scale of the $T$ effect is much smaller than that of the doping one). Another remarkable common feature of the spectral evolution under doping and $T$ variations is the existence of the crossover point $\omega_{\text {cr }}$ positioning near $E_{g}$ and dividing the VIS interband and the MIR intraband transitions: $\omega_{\mathrm{cr}}=1.55 \mathrm{eV}$ at doping and $\omega_{\mathrm{cr}}=1.4 \mathrm{eV}$ at $T$ variations (Fig. 4). It is seen that the overall integral spectral weight redistribution from VIS to MIR occurs on around the crossover point at which $\Delta \alpha_{\omega}=0$.

The above data on sensitivity of spectral functions to $T_{c}$ and on their different signs of the temperature dependences above and below the crossover point are in agreement with the results in [14]. In this work the ratio of reflection coefficients for the superconducting and the normal phases near $T_{c}$ was measured by applying the temperature modulation of HTSC samples. The data of the measurements are shown in Fig. 5 for a $\mathrm{BiCaCuO}$

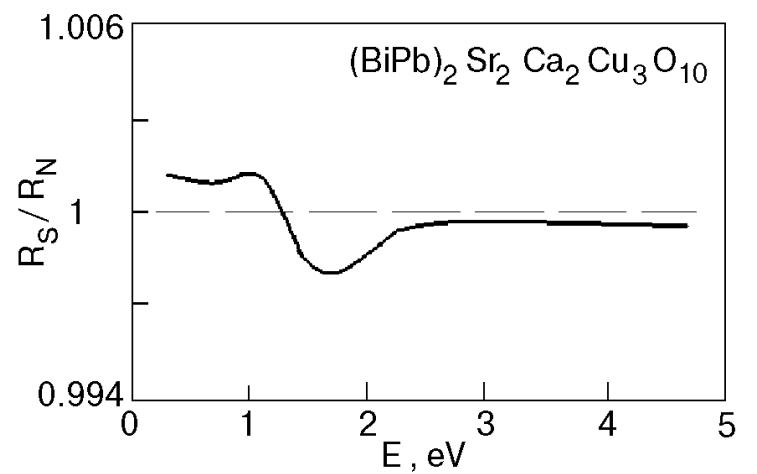

Fig. 5. The superconducting to normal-state reflectance ratio, $R_{s} / R_{n}$ of Bi-contained HTSC at $T=90 \mathrm{~K}\left(R_{s} / R_{n}\right.$ deviates significantly from unity at energies $0.5-2.5 \mathrm{eV}$ ) [14]. film. As is evident, the response of the reflection spectra to the SC transition is prominent for the $1.0-2.5 \mathrm{eV}$ region, within which there is an crossover point $(\hbar \omega \cong 1.4 \mathrm{eV})$. Since the temperature modulation range nearby $T_{c}$ was quite narrow $( \pm 5 \mathrm{~K})$, it is believed that the peculiarity nearby $T_{c}$ is jumplike in character. It should be noted that by the data on reflection shown in Fig. 5 the jump scale is $\Delta R / R \cong 0.3 \%$ while by our data on absorption (Fig. 4) it is equal to $\Delta \alpha / \alpha \cong 1-2 \%$.

The data shown suggest an idea of the $T$-induced correlation redistribution between VIS and MIR spectra and the strong coupling of oscillators at CT and MIR transitions. Our data are interesting because they were obtained at LT under the conditions where the charge transfer between $\mathrm{CuO}_{2}$ and $\mathrm{CuO}_{x}$ planes is hindered. It is known that with decrease in temperature down to the pseudogap state, the interaction between the $\mathrm{CuO}_{2}$ and the $\mathrm{CuO}_{x}$ planes is sharply diminished (there occurs a strengthening of the two-dimensionality of the $\mathrm{CuO}_{2}$ plane), as evidenced by the considerable reduction in the optical conductivity along the $c$ axis at frequencies of the scale of the pseudogap value $\hbar \omega<40 \mathrm{meV}$ [6]. It is obvious that this process reflects the freezing of charge transfer between the different planes of the YBCO cell.

So, there exists an optical response of all the spectral functions at frequencies hundreds times higher than the superconducting gap which is opened under the transition of HTSC systems to a superconducting state. It may be assumed that this nonconventional effect in HTSC is responsible for by a high ratio $\left(\Delta / \varepsilon_{F}\right)^{2} \cong 10^{-2}$ which determines the effect scale and is hundreds times more than that in conventional BCS materials. The optical response mechanism was considered theoretically from different viewpoints in Refs. 15, 16 and still remains debatable. Moreover, the sensitivity of spectral functions to $T_{c}$ occurs against the background of temperature-induced correlation mixture low- and high-frequency excitations. Therefore, the optical response suggests that either (i) superconditivity stimulates an extra additional correlation redistribution of excitations or (ii) high-frequency excitations of $1 \mathrm{eV}$ are directly involved in the mechanism of high- $T_{c}$ superconductivity.

\section{Photodoping at low temperatures}

One of the important questions of the optical spectroscopy of HTSC is the question of photoirradiation critical dose, $D_{0}$, and the threshold spectral energy, $\hbar \omega_{\text {th }}$, which determine permissible doses and energies of photoirradiation without persistent 


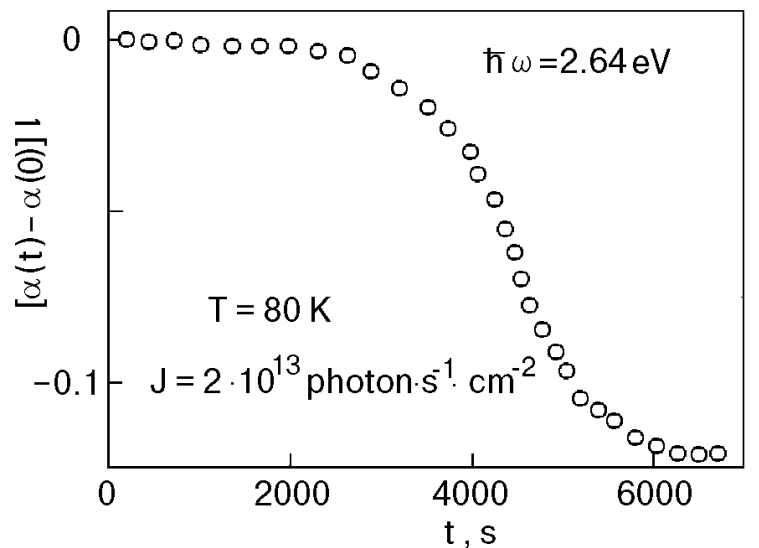

Fig. 6. Dose dependence of the absorption in YBCO with $x=0.35$ under irradiation and at measurements with $\hbar \omega=2.64 \mathrm{eV}$. At doses $D=J t>10^{17}$ photon $\mathrm{cm}^{-2}$ the sharp decrease of VIS absorption occurs due to photoinduced insulatormetal phase transition.

photoinduced effects in the lattice structure and in the current characteristics. It is known that a HTSC material is sensitive to exposure dose in VIS with the threshold spectral energy $\hbar \omega_{\text {th }}>\hbar \omega_{q}$ (in the region of the CT transitions) and with the doses $D>D_{0} \cong 10^{19-20}$ photon $/ \mathrm{cm}^{2}$. That is, using these conditions of photoirradiation at RT it becomes possible to observe an enhancement of $T_{c}$ in HTSC underdoped samples after photopumping $[5,17,18]$. The accepted explanation of the photoinduced conductivity is based on the assumption that the photoexcitation at RT causes the ordering of oxygen vacancies in the $\mathrm{CuO}_{x}$ chains that in turn leads to a subsequent transfer of electrons between the $\mathrm{CuO}_{2}$ and the $\mathrm{CuO}_{x}$ planes and the emergence of excess holes in $\mathrm{CuO}_{2}$ [17].

At the same time in some works it is found that the threshold dose of photoinduced effects become less by about two orders of magnitude if photopumping is carried out at low temperatures, $D_{0} \approx 10^{17}$ photon $/ \mathrm{cm}^{2}[9,19]$. The progressive increase of photodoping and the LT photoinduced phase transition of $\mathrm{Y}_{1} \mathrm{Ba}_{2} \mathrm{Cu}_{3} \mathrm{O}_{6.35}$ from the dielectric to a metallic state can be traced by the dose dependence of the absorption coefficient. Figure 6 shows the changes in the absorption coefficient as compared to the initial moment of irradiation with a light of $J=2 \cdot 10^{13}$ photon $\cdot \mathrm{s}^{-1} \cdot \mathrm{cm}^{-2}$ at $80 \mathrm{~K}$ of $\mathrm{Y}_{1} \mathrm{Ba}_{2} \mathrm{Cu}_{3} \mathrm{O}_{6+x}(x=0.35)$. As is evident, there occurs a sharp passage of the dependence $\Delta \alpha_{\omega}(t)$ to saturation at $D>J t \approx 10^{17}$ photon $\cdot \mathrm{cm}^{-2}$, suggesting the insulator-metal phase transition. It should be mentioned that the phase transition under LT photopumping follows the pattern of the second-kind phase transition. In Fig. 7 the process manifestation

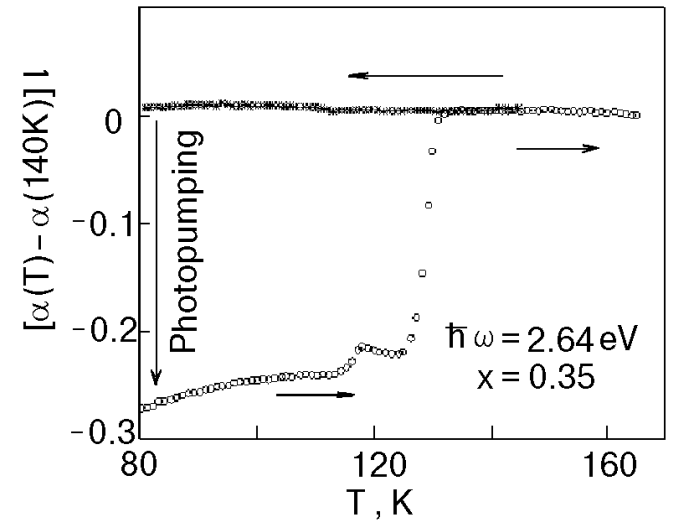

Fig. 7. Temperature dependences of the differential absorption coefficient in YBCO with $x=0.35$ before (the upper curve) and after (the lower curve) LT photopumping with $\hbar \omega=2.64 \mathrm{eV}, D=10^{17}$ photon $\cdot \mathrm{cm}^{-2}$.

of $\mathrm{YBaCuO}$ metallization under LT photopumping is seen from the temperature dependence of the absorption coefficient. The upper curve (on cooling the sample) corresponds to the dielectric state of $\mathrm{YBaCuO}$ with $x=0.35$ prior photopumping. As is evident, in this case the behavior of $\alpha(T)$ is temperature independent, which is typical of the insulator phase. After exposure of the sample to the light flux of $D=10^{17}$ photon $\cdot \mathrm{cm}^{-2}$ at $80 \mathrm{~K}$ and with subsequent increase in temperature, one can observe a temperature dependence of absorption typical of HTSC metals (compare with Fig. 2,b). At $T=135$ $\mathrm{K}$ a sharp reduction in the photopumped holes occurs due to their recombination with electrons so that the sample reverts to the original initial insulator state. The temperature of the reduction in the photopumped carriers (the threshold temperature) is dependent on doping of samples. The threshold temperature $T_{\mathrm{th}}=135 \mathrm{~K}$ was also observed in [4] by the decrease weakening of metallization on the curves $\alpha(T)$.

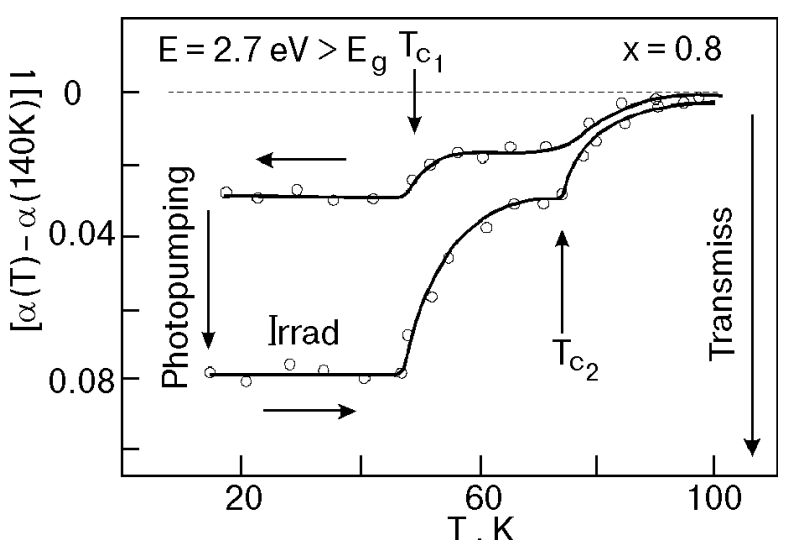

Fig. 8. Temperature dependences of the absorption in YBCO with $x=0.8$ before (the upper curve) and after (the lower curve) LT photopumping into SC phase with $\hbar \omega=2.7 \mathrm{eV}$, $D \geq 10^{17}$ photon $\cdot \mathrm{cm}^{-2}$. 
The effect of photodoping is also observed at immediate pumping into the superconducting phase. The experimental results for a two-phase sample with SC transitions at $T_{c 1}=50 \mathrm{~K}$ and $T_{c 2}=75 \mathrm{~K}$ are illustrated in Fig. 8. After the LT photopumping the sample is seen to become more transparent and with subsequent heating it returns to the initial state. The sharp decrease in the nonequilibrium concentration of photopumped holes is occurring at superconducting transition temperatures. We assume that the superconducting state is favorable to the retention of photopumped holes. As is seen from Fig. 9, the photopumping results in the correlation spectral weight redistribution between VIS and MIR, i.e., it acts like chemical doping. We can see a decrease in the integral absorption coefficient in $\mathrm{Y}_{1} \mathrm{Ba}_{2} \mathrm{Cu}_{3} \mathrm{O}_{6.8}$ after LT photopumping, i.e., due to the generation of nonequilibrium extra holes.

Our understanding of the process of persistent photodoping at LT is distinguished from the accepted ones at RT and is as follows. We think that the photoinduced holes appear in the valence band as a result of the charge-transfer excitations in the $\mathrm{CuO}_{2}$ plane with $\hbar \omega>E_{g}=1.6 \mathrm{eV}\left(\mathrm{Cu}^{2+} \mathrm{O}^{2+} \stackrel{\hbar \omega}{\rightarrow}\right.$ $\stackrel{\hbar \omega}{\rightarrow} \mathrm{Cu}^{+} \mathrm{O}^{-}$). Thus photopumping will be persistent if some obstacles to a reversible process exist. We suggest that a new process arises at LT photopumping that hampers the reversible recombination. This new process is considered in [20]. It is based on the concept of appropriate generation of low-temperature heavy quasi-particles-fluctuons. The physical concept of fluctuons was first introduced in the physics of solids by I.Lifshitz [21], and their phenomenological model was developed in [22]. In HTSC systems a hole fluctuon in the active $\mathrm{CuO}_{2}$ plane is easily generated through hole trapping by spin fluctuations inherent in the pseudogap state at $T<T^{*}$. In the $2 D$ case particularly deep fluctuon wells are generated with reduction in temperature, the well

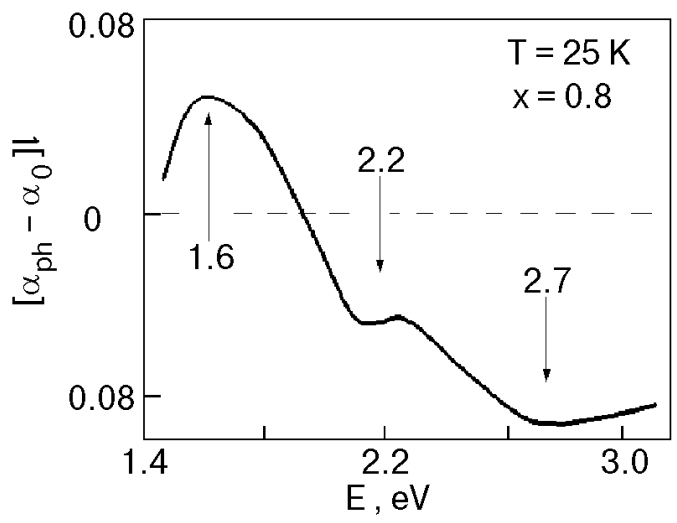

Fig. 9. The differential absorption spectra of YBCO with $x=0.8$ in the CT interband region of frequencies after LT photopumping. depth increasing as $T^{-2}$ [20]. It is the hole trapping by these spin fluctuons that results in the retention and accumulation of photoinduced holes. As the number of photoinduced holes increases, the fluctuon-fluctuon interaction determines a nonlinear process of photopumping (Fig. 6) and gives rise to an infinite metallic cluster following the pattern of the second-order phase transition.

So, the LT photopumping of HTSC at $T<T^{*} \approx$ $\approx 150-200 \mathrm{~K}$ (in the pseudogap state) with reduced threshold doses may be attributed to the effective hole trapping by spin fluctuations followed by generation of heavy fluctuons. It is likely that it is heavy fluctuons that determines a temperature- and photo-dependent addition to the spectral weight of the MIR region at LT, resulting in the correlation redistribution in the $\mathrm{CuO}_{2}$ plane.

\section{The LT relaxation in YBCO samples observed in optical spectroscopy}

Yet another low-temperature peculiarity in the behavior of spectral functions of HTSC at $T<T^{*}$ was observed with varying direction and velocity of $T$ scanning of the samples, namely, hysteresis effects in absorption and reflection. The hysteresis loop under cooling and heating of $\mathrm{YBaCuO}$ was mentioned in our former work for VIS absorption (Fig. 10) [12] and then subsequently the same effect was observed in measurements of MIR absorption (Fig. 2) and reflection (Fig. 11). As is evident from Figs. 10 and 11, as the samples are cooled, there occurs an accumulation of nonequilibrium that may be discarded (completely or partially) if the sample is held at LT for $\Delta t$. The set of our data suggests that the upper temperature boundary of instability is a temperature in the vicinity of the phase transition from the pseudogap

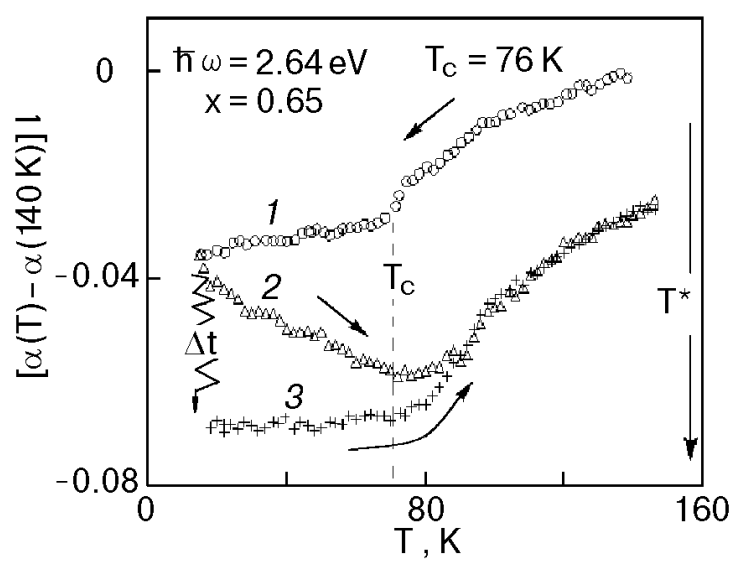

Fig. 10. Nonreversible effect in VIS absorption upon cooling and heating of the underdoped YBCO. Curves 1 and 2 were measured with uninterrupted cooling and heating. Curve 3 was measured after a long pause at $20 \mathrm{~K}(\Delta t \approx 40 \mathrm{~min}$ before heating). 


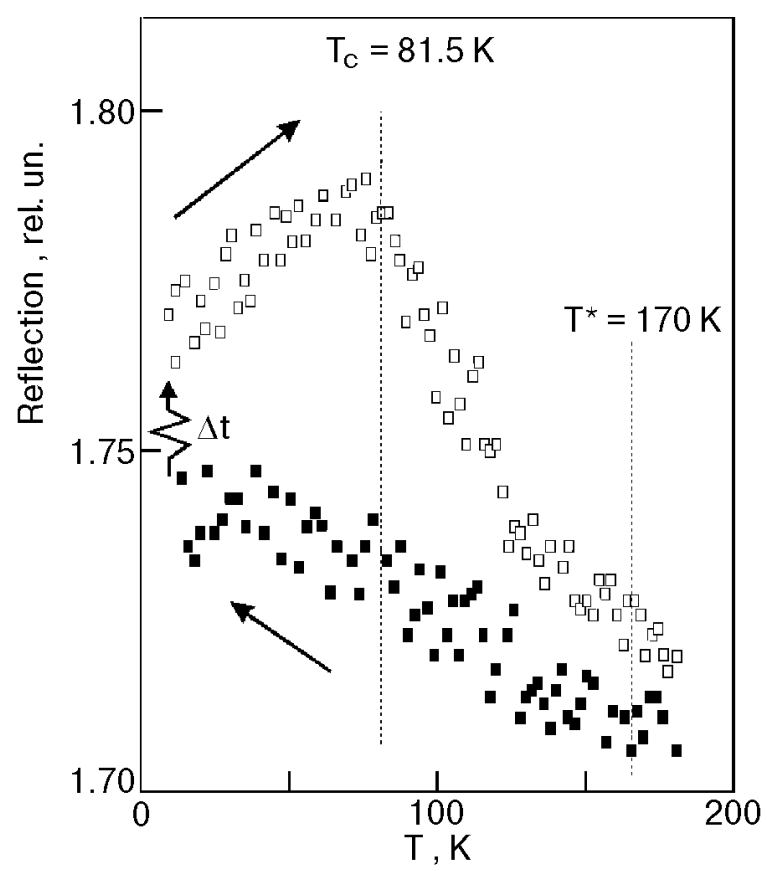

Fig. 11. The reflection spectra of $\mathrm{YBCO}$ in MIR region at $\hbar \omega=0.6 \mathrm{eV}$ upon cooling and heating.

state to states with disordered spins, $T^{*}$. The most pronounced hysteresis (electron-structure instability) is displayed by underdoped samples. With increasing doping, the loop area in the spectral function-temperature coordinates reduces, and the loop itself shifts towards lower temperatures. For samples of optimal doping, the hysteresis effects are minimum and may be observed with rather fast temperature cycling between the SC and $\mathrm{N}$ phases solely within the $T<T_{c}$ range. Generally speaking, the occurrence of hysteresis effects is associated with the existence of relaxation processes within a hysteresis loop temperature range. It is important to stress that the hysteresis loop in the MIR spectra is temperature inverse to that of the VIS spectral measurements. This means that the absorption (re-

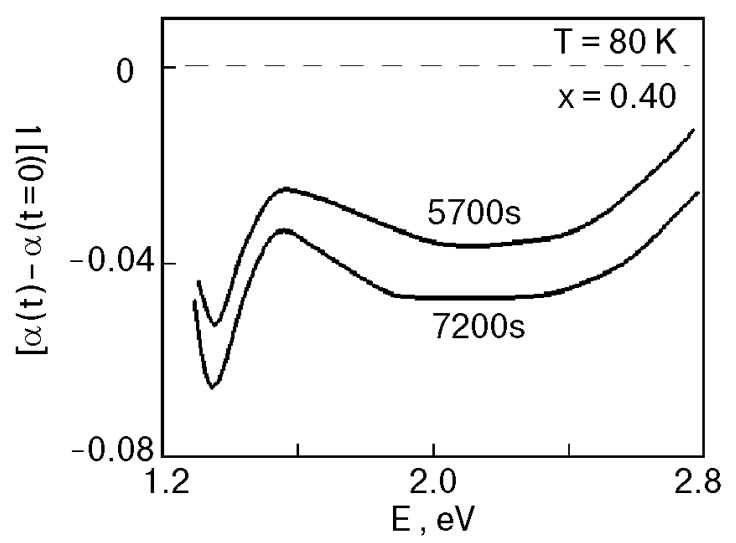

Fig. 12. The differential absorption spectra of $\mathrm{YBCO}$ with $x=0.4$ in CT interband region of frequencies at different time of relaxation after fast cooling. flection) coefficient undergoes changes in different directions in VIS and MIR during relaxation (Figs. 10, 11). All the experiments with instabilities of spectra were performed with low light doses $\left(D<D_{0}\right)$. We also carried out a special test-measurement with a dark window during relaxation and therefore we are convinced that the instabilities observed are not associated with the photoinduced effect. Note that the resistive measurements in different HTSC films exhibited no hysteresis effects with a loop span of several percents.

The differential spectra of VIS absorption of a sample with $x=0.4$ (in the vicinity of dielectricmetal transition) measured for two relaxation times $\left(t_{1}=5700 \mathrm{~s}\right.$ and $\left.t_{2}=7200 \mathrm{~s}\right)$ after cooling from 220 to $88 \mathrm{~K}$ are plotted in Fig. 12. It can be seen that the absorption decreases through the whole VIS region, i.e., the integral bleaching of the CT transitions occurs. The characteristic time of LT-relaxation could be estimated as $\tau \cong(1-2) \cdot 10^{3} \mathrm{~s}$. Time modifications in the absorption and reflection spectra are also observed in the MIR region after rapid cooling down to a certain temperature. These modifications occur with increasing both the integral MIR absorption and the MIR reflection, i.e., during relaxation the MIR and VIS optical characteristics undergo changes in different directions just as it occurs under chemical or photodoping. At the same time, in the near UV region for $\hbar \omega=4.1 \mathrm{eV}$, in which there are optical transitions $(3 d-4 p)$ on local centers of $\mathrm{Cu}^{+}$, one cannot observe relaxation effects in absorption at LT, i.e., the concentration of monovalent $\mathrm{Cu}^{+}$ions in the chain structure of $\mathrm{CuO}_{x}$ remains unchanged after temperature variations in the $T<T^{*}$ region (Fig. 13).

Figure 14 presents relaxation curves for the underdoped metal film of $\mathrm{Y}_{1} \mathrm{Ba}_{2} \mathrm{Cu}_{3} \mathrm{O}_{6+x}$ with $x=0.7$ at $T_{c}=70 \mathrm{~K}$. The data for different temperatures

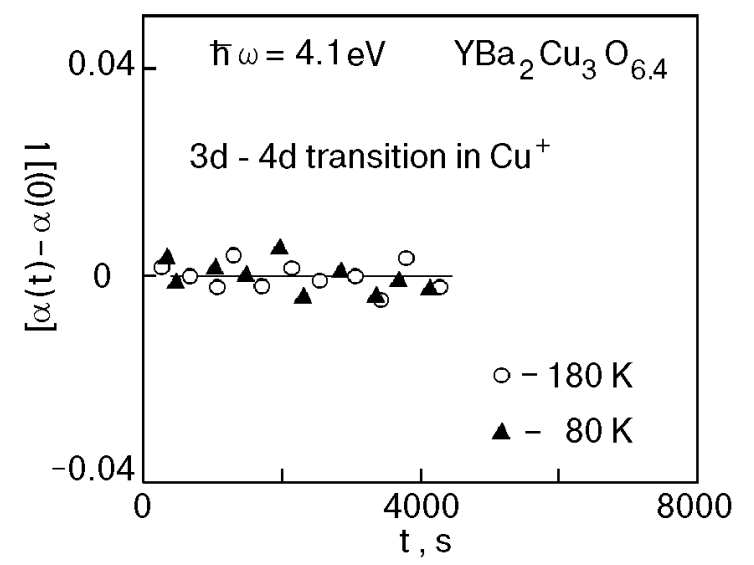

Fig. 13. Time dependence of the differential absorption coefficient at $\hbar \omega=4.1 \mathrm{eV}$ after fast cooling of YBCO from $220 \mathrm{~K}$ to $180 \mathrm{~K}(\mathrm{O})$ and to $80 \mathrm{~K}(\mathbf{\Delta})$. 


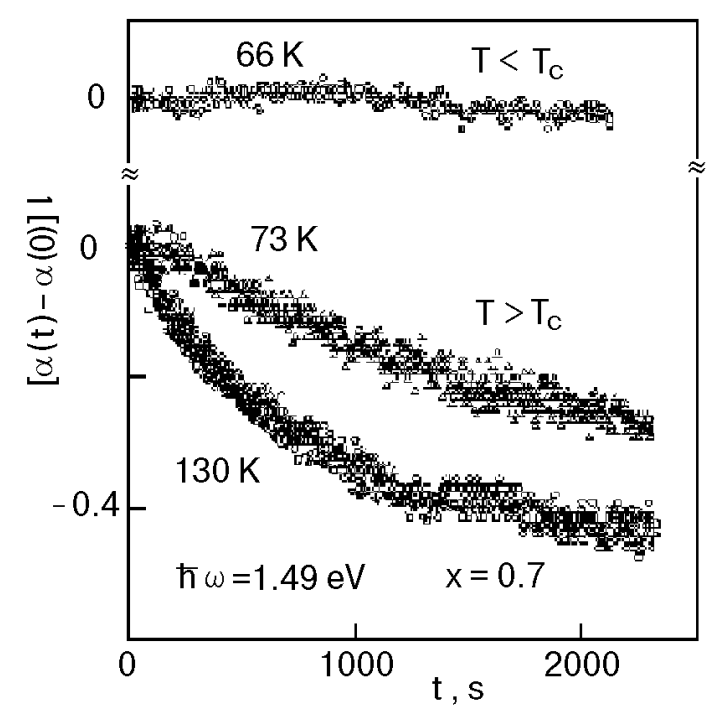

Fig. 14. Time dependences of the differential absorption coefficient at $\hbar \omega=1.49 \mathrm{eV}$ in process of relaxation after cooling to different temperatures $T>T_{c}=70 \mathrm{~K}$ and $T<T_{c}$.

were obtained in sequential measurements upon cooling from $250 \mathrm{~K}$. Differential absorption $\Delta \alpha l=$ $=\left(\alpha^{f}-\alpha^{i}\right)$ vs. $T$ is plotted relative to the initial moment of temperature stabilization $\left(T_{i}\right)$ of $\alpha_{i}(t=0)$. After each previous case where the curve $(t)$ reached saturation at a certain temperature, the temperature was quickly decreased to a next level and relaxation was measured once again and so on. On this slow cooling, upon reaching the equilibrium state (saturation) one can see that relaxation is absent below $T<T_{c}$.

It seems to be likely that the $T$-induced time instability revealed in VIS optical absorption of CT transitions, which belong to the $\mathrm{CuO}_{2}$ plane, reflects the inherent electron (spin)-structure relaxation in the active $\mathrm{CuO}_{2}$ plane at LT variations. In the course of this relaxation the spectral weight redistribution from the VIS to the MIR region occurs. If we accept this assumption, two questions arise immediately: 1) what is the nature of the electron-structure relaxation at low temperatures? 2 ) what is the reason why the LT relaxation is not so clearly observed in experiments on the dc resistivity?

Let us remember that the $T$-induced structure relaxation resulting in ortho-ordering and variations of spectral functions of HTSC samples after fast cooling from high temperature $\left(400{ }^{\circ} \mathrm{C}\right)$ down to $\mathrm{RT}$ are described in a series of papers [3,23]. The commonly accepted model of RT relaxation of an order parameter is based on the mechanism of diffusion rearrangement of oxygen vacancies making the copper-oxygen chain $(\mathrm{Cu}-\mathrm{O}-\mathrm{Cu})$ along the $b$-axis in $\mathrm{CuO}_{x}$ longer and following the charge transfer between the $\mathrm{CuO}_{x}$ and $\mathrm{CuO}_{2}$ planes. One of the arguments in favor of this model was the evidence for the variations of the $\mathrm{Cu}^{+}$concentration (the variations observed in the optical reflection of the $(3 d-4 d)$ transitions of the $\mathrm{Cu}^{+}$ions at $4.1 \mathrm{eV}$ vs. time [3]).

In contrast to the RT relaxation of the spectral functions, the LT relaxation of the CT interband and MIR intraband spectral weights is not accompanied by variations of the $\mathrm{Cu}^{+}$concentration (Fig. 13). This result is against the electron-structure rearrangement in $\mathrm{CuO}_{x}$, where the $\mathrm{Cu}^{+}$ions reside, and the evidences that the $\mathrm{LT}$ relaxation occurs exactly in the $\mathrm{CuO}_{2}$ plane without any charge transfer between the planes (the interplane charge transfer is likely to be hindered at LT). We assume that the most reliable explanation of the LT relaxation of the spectral functions in VIS and MIR is the temperature-induced spin structure rearrangement in the $\mathrm{Cu}^{+}$plane due to the formation of hole-spin fluctuons at LT. Strong influence of the system prehistory and its cooling rate on the LT state and long relaxation times in underdoped HTSC suggest that these are similar to irreversible effects in nonergodic systems with competing interactions (for example, to structural or spin glasses [24]). From this viewpoint, the transition to the pseudogap state at $T^{*}$ may be considered as a transition to a nonergodic system where ordering regions alternate with disordering ones. In the fluctuon model the ordering occurs within a region round a hole trapped by spins. Note also that the concept of holes trapped in a spin bag [25] as well as the concept of holes trapped in the «string» potential created by AF background [26], or the model of stripes, that is the subject of wide speculation [27], are not dramatically different from our pattern of hole-spin fluctuons.

Thus, we think it quite reasonable to associate the LT instability of optical spectra and the effective photodoping at low temperatures with two processes - the hole trapping by spin fluctuations at low temperature and the induced spectral weight redistribution from VIS to MIR (due to strong correlation).

In parallel with the fluctuon domains where the trapped heavy holes reside, there exists a current network for light holes. Therefore, the light and heavy holes are in dynamic equilibrium resulting from the competition between the superexchange energy lost near a trapped hole and its kinetic energy. The spectral weight of intraband transitions is distributed between the contributions from light 
and heavy carriers. Note that in the MIR region the contribution from heavy holes is dominant while in the far low-frequency region $(\omega \rightarrow 0)$ the contribution from light holes is prevalent. The measurements of HTSC sample resistivity $(\omega \rightarrow 0)$ reveal mainly the contribution of light carriers not related to the spin-structure relaxation. d.c. conductivity $\sigma(0)$ is defined by plasma frequency $\omega_{p}^{2} \sim n_{L}(T, t)$ and damping $\Gamma(T, t)$ (where $n_{L}$ is the number of light carriers): $\sigma(0) \sim n / \Gamma$. The relative changes $\Delta \sigma / \sigma$ in the experiments with varying $T$ (or due to relaxation) are $\Delta \sigma / \sigma=\Delta n / n-\Delta \Gamma / \Gamma$. As is evident from the optical measurements, $\Delta n / n \sim \Delta \alpha / \alpha \simeq 5 \%$. At the same time $\Delta \Gamma / \Gamma \sim \Delta T / T$ and amounts to $50 \%$. Therefore, unlike the optical measurements, the resistive ones do not sense relaxation and temperature variations associated with the number of carriers and show mainly the $T$ variations in damping of light carriers.

So, the optical data reveal the dramatic effects of nonequilibrium inherent in HTSC systems after their fast cooling to the pseudogap state region below $T^{*}$. The effects are most pronounced for underdoped samples and much weaker for the optimal doping regime. Generally speaking, the high nonequilibrium resulted from the competitive interactions occurring in the HTSC system which bring two types of carriers (holes) with different spin surrounding into dynamic coexistence: light (coherent) holes and heavy holes trapped in a spin bag. As a result, the optical conductivity is determined by two contributions - a coherent part at the expense of Drude-like light carriers and a noncoherent one at the expense of heavy hole fluctuons. The optical relaxation observed arises from the self-consistent hole trapping by spin fluctuations and the subsequent spin ordering inside a fluctuon. In this case the ordering time is supposed to be $\tau \simeq \exp (a \sqrt{N})$, where $N>>1$, the number of spins in a fluctuon, may be rather high.

\section{Conclusion}

After the discovery of high- $T_{c}$ superconductivity a great number of works were concentrated on the problem of adequate description of the behavior of electrons in anisotropic cuprates. For the analysis of the above optical results we used the consequences of the theoretical concepts developed in a series of theoretical works as a basis. These works are directly or indirectly connected with a broader Hubbard-like model of strongly correlated $2 D$ electrons submerged in spin media [26, 28-33]. One of the important general results of these theories is that the behavior of holes in the AF background is accounted for by the competition between the superexchange energy lost near a hole and its kinetic energy. Thus, the Hubbard-like model evidences that there is a dynamical coexistence of mobile (coherent) holes and heavy holes trapped in a spin bag. The impressive calculations of the temperature evolution of DOS in terms of the Hubbard model are carried out in [33]. Away from half-filling of a lower Hubbard band a sharp resonance peak appears near the chemical potential. As the doping is increased the resonance width also increases and it starts to merge with the lower Hubbard band. For the overdoping regime, both low-energy peaks are indistinguishable, implying that the system has become an uncorrelated ordinary metal. The narrow peak in DOS evolves like the MIR band in the optical spectra. The narrow peak appears to be strongly temperature dependent, thus it is pronounced only in the region of low temperatures and within a regime that extends from underdoping to optical doping.

Recent low-temperature experimental and theoretical works have made it clear that in HTSC materials there appears a state below $T<T^{*}$ that is precursory to superconductivity. The temperature $T^{*}$ coincides with the temperature of the pseudogap opening with spin singlet ordering. This LT precursory region turns out to be optically active in the absorption and reflection spectra. Using the basic consequences of the theory of correlated electrons and the optical experimental findings, we propose the following principal ansatze:

1. The consistent concept of strongly correlated hole excitations interacting with spin fluctuations in the $\mathrm{CuO}_{2}$ plane is attractive for understanding and interpreting the anomalous optical effects in HTSC at LT having no analogue with conventional BCS superconductors (the sensitivity to $T_{c}$, the $T$ induced spectral weight redistribution, the LT relaxation of optical functions, the LT-enhanced photodoping).

2. A nontrivial consequence of the correlation model in the intermediate regime is the coexistence of light (Drude-like) and heavy (hole-spin-fluctuons) holes, the latter being generated in the LT region below the temperature of spin ordering (opening spin gap). Thus, the high- $T_{c}$ superconductive scenario is realized in dynamical two-component systems of carriers.

3. Subsystems of light and heavy carriers are in dynamical equilibrium upon doping or $T$ variations. If the doping of HTSC is increased the localization effects are weakened, while if the temperature is decreased, the localization effects become stronger 
and the density of heavy trapped holes increases. Thus, the temperature serves as fine tuning for high- $T_{c}$ superconductivity.

\section{Acknowledgments}

We are pleased to express our deep gratitude to Prof. G. Saemann-Ischenko and his colleagues for their useful scientific cooperation and preparation of HTSC samples for our optical measurements. We are grateful to Prof. H. Kinder and Dr. P. Berberich for providing the samples for the MIR measurement. The authors wish to express their thanks to S. Uyutnov for his help during optical measurenments. Some of results of this work were delivered at the NATO-ASI Conference «Materials Science, Fundamental Properties and Future Electronic Applications of High- $T_{c}$ Superconductors» (September, 1998).

1. S. Uchida, I. Ido, H. Takagi, T. Arima, Y. Tokura, and S. Tojima, Phys. Rev. B43, 7942 (1991).

2. S. L. Cooper, D. Reznik, A. Kotz, M. A. Karlow, R. Liu, M. V. Klein, and W. C. Lee, Phys. Rev. B47, 8233 (1993).

3. J. Kircher, M. Cardona, A. Zibold, K. Widder, H. P. Geserich, J. Giapintzanis, D. M. Ginsberg, B. W. Veal, and A. P. Paulikas, Phys. Rev. B48, 9684 (1993).

4. G. Yu, C. H. Lee, D. Mihailovic, A. J. Heeger, C. Fincher, N. Herron, and E. M. McCarron, Phys. Rev. B48, 7545 (1993).

5. V. Kudinov, A. Kirilyuk, N. Kreines, R. Laiha, and E. Lahderanta, Phys. Lett. A151, 358 (1990).

6. A. V. Puchkov, D. N. Basov, and T. J. Timusk, J.Phys. Condens. Matter 8, 10049 (1996).

7. C. M. Varma, Int. J. Mod. Phys. B3, 2083 (1989).

8. I. Fugol and V. Samovarov, in: Studies of HTSC ( $A d$ vances in Research and Applications), v. 22 A. Narlikar (ed), Nova Science Commack, New York (1997).

9. I. Fugol, G. Saemann-Ischenko, V. Samovarov, Yu. Rybalko, V. Zhuravlev, Y. Strobel, B. Holzapfel, and P. Berberich, Solid. State Commun. 80, 201 (1991).

10. H. L. Dewing and E. K. H. Salje, Supercond. Sci. Technol. 5, 50 (1992); J. Solid State Chem. 100, 363 (1992).
11. C. H. Ruscher, M. Gotte, B. Schmidt, C. Quitmann, and G. Guntherodt, Physica C204, 30 (1992).

12. I. Fugol, V. Samovarov, A. Ratner, V. Zhuravlev, and G. Saemann-Ischenko, Solid State Commun. 86, 385 (1993); Physica C216, 391 (1993).

13. I. Fugol, C. Politis, A. Ratner, V. Samovarov, and V. Zhuravlev, J. Lumin. 62, 291 (1994).

14. H. J. Holcomb, C. L. Perry, J. P. Collman, and W. A. Little, Phys. Rev. B53, 6734 (1996).

15. J. E. Hirsch, Physica C199, 305 (1992); ibid. 201, 347 (1992).

16. A. S. Alexandrov, A. M. Bratkovshy, N. F. Mott, and E. K. H. Salje, Physica C215, 359 (1993).

17. E. Osquiquil, M. Maenhoudt, and B. Wayts, Phys. Rev. B49, 3675 (1994)

18. V. M. Dmitriev, V. V. Eremenko, I. S. Kachur, V. G. Piryatinskaya, O. R. Prikhod'ko, A. M. Ratner, E. V. Khristenko, and V. V. Shapiro, Fiz. Nizk. Temp. 21, 219 (1995), [Low Temp. Phys. 21, 168 (1995)].

19. G. Yu, C. H. Lee, A. J. Heeger, N. Herron, E. M. McCarron, and Lin Cong, Phys. Rev. B45, 4964 (1992).

20. L. Kukuschkin, V. Samovarov, M. Libin, and I. Fugol, Fiz. Nizk. Temp. 22, 375 (1996) [Low Temp. Phys. 22, 290 (1996)].

21. I. M. Lifshitz and S. Gredeskul, Zh. Eksp. Teor. Fiz. 57 , 2209 (1969) [Soviet Phys. JETP 30, 1197 (1969)].

22. M. Krivoglaz, Uspekhi. Fiz. Nauk 111, 617 (1973) [Sov. Phys. Uspekhi 16, 856 (1974)].

23. H. Shaked, J. Jorgensen, B. Hunter, R. Hittermen, A. Paulikas, and B. Veal, Phys. Rev. B51, 547 (1995).

24. S. L. Ginzberg, in: Irreversible Phenomena of Spin Glasses, Nauka, Moscow (1989).

25. J. R. Schrieffer, X.-G. Wen, and S.-C. Zhang, Phys. Rev. B39, 11663 (1989).

26. E. Dagotto, Rev. Mod. Phys. 66, 763 (1994)

27. B. Levi, Physics Today, June, 19 (1998).

28. H. Eskes, M. Meinders, and G. Sawatzky, Phys. Rev. Lett. 67, 1035 (1991).

29. B. Stojkovic and D. Pines, Phys. Rev. B55, 8575 (1997).

30. S. M. Quinlan, P. J. Hirschfeld, and D. J. Scalapino, Phys. Rev. B53, 8575 (1996).

31. A. Sherman and M. Schreiber, Phys. Rev. B50, 12887 (1994).

32. V. M. Loktev, Fiz. Nizk. Temp. 22, 3 (1996) [Low Temp. Phys. 22, 1 (1996)].

33. Th. Pruschke, M. Jarrell, and J. K. Freerecks, Adv. Phys. 44, 187 (1995) 